\title{
Robert K. Merton, Erving Goffman, y el recurso del rol
}

Pablo De Grande ${ }^{1}$

Universidad del Salvador

\section{Tipo de trabajo: Artículo}

Material original autorizado para su primer publicación en el Journal de Ciencias Sociales, Revista Académica de la Facultad de Ciencias Sociales de la Universidad de Palermo

Recibido 17-6-2014

Aprobado 7-10-2014

\section{Resumen}

El término 'rol', o la idea más general de que un grupo de personas cumplen unos papeles más o menos conocidos y definidos, atraviesa el sentido común, así como también se presenta en proposiciones teóricas e investigaciones aplicadas en sociología. En este artículo se propone retomar algunos aportes de Robert K. Merton y Erving Goffman en torno a la relación entre individuos y ubicaciones sociales relativamente estandarizadas e institucionalizadas. A través de estos autores se propone reflejar la preocupación de los mismos por comprender la relación de los roles entre sí y de los roles con las personas que los desempeñan, de un modo que evite una representación de dichas relaciones como campos completamente coherentes, consistentes o libres de conflicto.

Palabras clave: sistema de papeles, performance, ritualismo

\begin{abstract}
The idea that a group of people may be actually playing a set of predefined roles belongs to common sense as well as it appears in theoretical developments and applied research in sociology. However, it's not possible to think of them as a complete and systematic theory of roles. This article elaborate on the contributions of Robert K. Merton and Erving Goffman about the relationship between individuals and social positions somewhat standardized and institutionalized. Merton and Goffman investigated the relationship among different roles in a system of roles, as well as the relationship between a role and the person who performs it, looking to reflect the complexity of such relations, as fields never completely coherent, consistent or free of conflicts.
\end{abstract}

Keywords: systems of roles, performance, ritualism

\footnotetext{
${ }^{1}$ Profesor - investigador Instituto de Investigación en Ciencias Sociales de la Universidad del Salvador, Argentina. pablodg@gmail.com
} 


\section{Introducción}

Este trabajo tiene por objetivo comentar algunas características de los abordajes teóricos de Robert K. Merton y Erving Goffman, relacionando la obra de ambos autores en el contexto de la sociología norteamericana de segunda mitad del siglo XX. La selección del corpus comentado incluye tres obras: La presentación de la persona en la vida cotidiana, de Erving Goffman (1971), publicado originalmente en 1959; y dos artículos de Robert K. Merton, publicados en 1949 en su libro Teoría y estructuras sociales (con una edición revisada en 1957), titulados Funciones manifiestas y latentes y Estructura social y anomia (1995a y 1995b).

El interés por relacionar estos autores surge del propósito de conectar la producción de dos obras temporal y culturalmente cercanas, vinculándolas conceptualmente por la relevancia compartida del concepto de rol. La hipótesis de este trabajo es que existen elementos a través de los cuales es posible colocar a estos autores en un espacio común de producción teórica que enfatiza la necesidad de hacer visible los aspectos complejos -contradictorios, ambiguos, solapados- de los sistemas de roles y sus funcionamientos concretos.

\section{Presentaciones breves}

\section{Erving Goffman}

Nació en Alberta, Canadá, en 1922. Estudió sociología en la Universidad de Toronto, donde se mantuvo en proximidad con el campo de la antropología (Ritzer, 2002, p. 280).

Al haber realizado su tesis doctoral en Chicago, se lo asocia con frecuencia a la Escuela de Chicago y al interaccionismo simbólico. Sin embargo, según Ritzer, cabe señalar que son más frecuentes en sus citas tempranas los trabajos de antropología social, siendo las citas a interaccionistas más escasas y -cuando las hay-con actitud crítica.

Murió en 1982, en la cima de su reconocimiento intelectual (Collins, 1986), siendo presidente de la American Sociological Association, en la que no alcanzó a realizar su discurso anual inaugural por causa de su deteriorada salud.

\section{Robert K. Merton}

Nació en Filadelfia, Estados Unidos, en 1910. Estudió sociología en la Universidad de Temple y comenzó a trabajar como graduado en la universidad de Harvard en 1932 (Crothers, 1987, p. 23). En 1941 pasa a la universidad de Columbia (Nueva York), desarrollando una trayectoria académica destacada, siendo presidente de la American Sociological Association entre 1956 y 1957, de la Eastern Sociological Society en 1968 y 1969, de la Sociological Research Association en 1968 y de la Society for Social Studies of Science en 1975 y 1976. Desarrolló en sociología varios términos de uso corriente, como el de profecía auto-cumplida y el de 'teoría de alcance medio', entre otros. Entre sus principales colaboraciones e intercambios académicos se destacan sus durables intercambios con P. Sorokin en Harvard, y con P. Lazarsfeld a lo largo de varias décadas en Columbia. 
Murió en febrero de 2003 (Cole, 2004, p. 829), tras publicar decenas de artículos que abordaron tanto problemas de teoría como preocupaciones metodológicas y problemas empíricos de sociología aplicada.

\section{Síntesis del corpus}

\section{Erving Goffman}

En La presentación de la persona en la vida cotidiana, Goffman introduce una diversidad de ejemplos, herramientas y argumentos para sostener la noción de que las interacciones cotidianas de los individuos deben ser vistas como actos complejos de negociación, de control, de autocontrol y de adaptación, y no ya como simples diálogos o intercambios.

Estos procesos y mecanismos que el autor presenta y pone de relieve a lo largo del libro cuestionan las nociones de espontaneidad, sinceridad y transparencia tal como los mismos sujetos en el sentido común de la cultura angloamericana suelen representarlo. Estas categorías son reemplazadas por una visión según la cual toda expresión -independientemente de la voluntad de transparencia con la que sea efectuada- debe ser comprendida bajo la tensión de las miradas, intereses y expectativas recíprocamente intervinientes de los actores. Estos actores proyectan sobre los demás sus ideas de roles, atributos y actitudes que esperan, a la vez que intentan controlar las percepciones que los demás se hacen de ellos, todo de un modo 'automático', de un modo no usualmente razonado pero sistemáticamente operante en la conducta social².

A partir de una propuesta situada en el nivel de la estructuración subjetiva de las interacciones cotidianas, es posible reconocer cuatro grandes temas a lo largo de la obra. En los capítulos 1 y 3 (a) se presenta la concepción fundante en Goffman de las 'actuaciones' (performances). En el capítulo 2 (b), se hace un tratamiento explícito del funcionamiento de dichas actuaciones por parte de grupos, es decir, cómo un conjunto de personas operan coordinadamente al compartir pretensiones similares de verosimilitud para una cierta realidad performativa. En los capítulos 4 y 5 (c) se plantea la cuestión de la consistencia de los roles y actuaciones, para lo cual se explica el modo en que opera la administración por parte de los actores de las diferentes escenas que deben sostener. En el capítulo 6 (d) se detallan algunas técnicas y atributos que los sujetos deben manejar y disponer para poder realizar de forma efectiva sus pretensiones de control sobre sus actuaciones. A continuación, se detallan brevemente algunos de los elementos salientes que el autor reúne para cada uno de estos temas del libro.

(a) El concepto de 'actuación' (performance) refiere a la actividad que un individuo hace frente otros, en la cual mantiene un cierto grado de influencia sobre ellos. Dentro de la situación de actuación, distingue a su vez entre regiones, destacándose la 'fachada' (front region) y la región posterior (back region) o trasfondo escénico (backstage). La fachada objetiva aquello que los destinatarios

\footnotetext{
2 Así, el sujeto "puede estar sinceramente convencido de que la impresión de realidad que pone en escena es la verdadera realidad. Cuando su público también se convence de la representación que él ofrece -y este parece ser el caso típico-, entonces, al menos en principio, solo el sociólogo o los resentidos sociales abrigaran dudas acerca de la 'realidad' de lo que se presenta" (Goffman, 1971, p. 29).
} 
de la presentación ven, existiendo una separación entre ella y el backstage de las actuaciones. Esta separación divide públicos diferenciados, que tienen o no permitido ver una u otra regiones. Sea que un sujeto esté a punto de salir con su pareja, ensaye una presentación laboral o esté por recibir a su jefe para cenar, en todos los casos las acciones, recursos y recaudos que se toman en tanto preparación previa a la actuación muestran matices y acciones específicos que organizan el desarrollo de estas actividades. Asimismo, destaca el autor el carácter más eminentemente técnico de las tareas de preparación, por oposición al carácter principalmente expresivo / dramático de la puesta en escena propiamente dicha.

(b) La introducción de la problemática de los equipos (o 'elencos', pero también haciendo del auditorio un equipo) enriquece notablemente el primer concepto de 'actuación'. Por medio del funcionamiento cooperativo de varias personas en una situación dada, el autor resalta que por una parte las actuaciones (como puestas en juego de una verdad construida) suelen apoyarse en el desempeño en escena de más de una persona, lo que es especialmente crucial cuando se busca sostener una realidad que por definición supone una pluralidad de sujetos en roles o bien semejantes o bien complementarios (la imagen de una familia feliz, de una empresa funcionando laboriosamente, de un grupo de deportistas motivados hacia una meta, etc.). Pero, por otra parte -no menos importante-, se ilustra por este medio el hecho de que las actuaciones no son exclusivamente la presentación de la personalidad o interioridad de los sujetos. Por el contrario, las actuaciones permiten dar realidad a construcciones sociales que poco dependen de los rasgos de los sujetos, y que para realizarse y sostenerse precisan de personas consustanciadas con su puesta en escena.

(c) La noción de regiones (front y backstage) sugería ya la existencia de espacios separados cuya interacción imprevista podía provocar efectos no deseados. Estas discrepancias remiten en la obra a un tratamiento particular, realizada en torno al problema del control sobre la información, es decir, acciones y situaciones en las que los actuantes deben prestar atención a que cierta 'información destructiva' no llegue a su auditorio (la administración de los 'secretos', pero también de la producción de nueva información poco conveniente). Asimismo, hay conversaciones que, sin ser secretos guardados, se desarrollaron separadamente, tal como lo son las conversaciones detrás de escena. De esta forma, los profesores pueden tomarse ciertas licencias al hablar de sus alumnos, o los cocineros de sus comensales, lo que reporta la función adicional de reforzar la identidad y unidad del equipo actuante.

(d) Por 'el arte de manejar las impresiones' se alude a diferentes aspectos de la fragilidad de las actuaciones. De esta forma, se comenta cómo una intrusión inoportuna o un gesto equivocado puede producir situaciones incómodas para los involucrados. Resalta también la importancia de la 'lealtad', entendida en el sentido amplio de guardar adhesión a los contenidos morales, el cuidado de los secretos, y todo aquello que pueda minar las pretensiones de verosimilitud de las actuaciones de los grupos ante los demás, así como de la disciplina que los actuantes deben sostener a lo largo de sus interacciones.

Por último, en las conclusiones, sitúa su trabajo desde algunos aspectos teóricos, no necesariamente hechos explícitos en la obra. En este sentido, Goffman introduce el concepto de marco de referencia, apelando a él para sugerir su esquema de análisis, donde actores agrupados en equipos despliegan 
sus estrategias dramáticas, como una herramienta formal y abstracta de abordar un abanico amplio de situaciones de interacción. Más tardíamente Goffman utilizará este término en forma más desarrollada para remitir a los registros de saberes y prácticas que los actores deben reconocer para poder interpretar y actuar en un espacio determinado (Goffman, 1974).

También en el cierre se hace explícito el interés en su abordaje por conectar los niveles de análisis de la personalidad con aquellos de la interacción y el de la sociedad. Finalmente, el autor comenta las implicancias que encuentra para su análisis en la percepción de lo que se entiende por 'sí mismo', integrando en el individuo la doble existencia de actuante y de personaje.

\section{Robert K. Merton}

En Estructura social y anomia, Robert K. Merton (1995a) critica en primer lugar el modelo explicativo por el cual el orden social vendría a controlar unos impulsos biológicos originarios, inmanentes a todo ser humano. Según esta concepción, la estructura social supondría una reducción del sujeto, contrapuesta a la naturaleza plena, originaria, biológica, del hombre.

La propuesta del artículo es ilustrar sobre la búsqueda de presiones sociales que incentiven al disconformismo y a la desviación, de modo de poder realizar a partir de este esquema caracterizaciones o explicaciones sociológicas que permitan disociar 'desviación social' de 'patología psicológica'.

Para tratar el tema, define dos elementos centrales, haciendo uso de una distinción bastante clásica, a saber, la de medios y fines.

Sobre los fines, se interesa en aquellos que se constituyen en 'objetivos legítimos', o 'intereses estructuralmente definidos', que son los logros que los sujetos encuentran como disponibles para fijarse como metas individuales en sus vidas. Estos objetivos se encuentran ordenados en una jerarquía que establece diferentes niveles valorativos para los posibles logros socialmente alcanzables.

Los 'medios' refieren a las formas institucionalizadas en que es esperable que se logren dichos fines, también encuadrados en el concepto de 'legitimidad' como marco de posibilidad y aceptabilidad de ciertos cursos de acción por sobre otros.

Presentados estos elementos, Merton plantea el problema del carácter inestable (o al menos no garantizado) del equilibro entre estos dos aspectos de la estructura social. $O$, en otros términos, ¿qué sucede cuando los medios admisibles no conducen a los fines legitimados?

Cuando cierta sociedad ejerce una gran presión con relación a objetivos específicos sin preocuparse sin embargo por asegurar procedimientos valorativamente aceptados (por oposición a 'técnicamente válidos') para su consecución, comienza a inducir a sus miembros a perseguir tales fines sin interés por la legitimidad de sus medios, en dirección hacia el escenario caracterizado por Durkheim como de 'anomia' (Merton, 1995a, p. 213).

Luego, presenta ejemplos en los cuales sitúa a la sociedad estadounidense ${ }^{3}$ como sumergida en una

${ }^{3}$ La traducción afirma 'norteamericana', pero la versión inglesa de un artículo similar (aunque no idéntico) reeditado en On 
diversidad de presiones en función de perseguir en su conjunto unas únicas metas de prosperidad económica, destino que, sin embargo, no es posible en términos prácticos (y mucho menos legales) lograr para un porcentaje importante de su población. La riqueza económica como único punto de llegada aceptable para sus miembros provoca desajustes que Merton intenta clasificar. A partir de estos ejes, establece las categorías de adaptación individual de (a) conformidad, (b) innovación, (c) ritualismo, $(\mathrm{d})$ retraimiento y $(\mathrm{e})$ rebelión. Estas se caracterizan por:

(a) Conformidad: reúne aquellos sujetos cuya conducta típica coincide con las normas y valores compartidos y generalizados de la sociedad, y según Merton es condición para el funcionamiento de una sociedad que este tipo de adaptación sea el mayoritario.

(b) Innovación: este segundo tipo reúne a los sujetos que, al restar importancia a la legalidad o institucionalidad de sus medios, procuran alcanzar fines socialmente establecidos por medios que están 'más allá de las costumbres' (1995a, p. 220). Con esto incluye a conductas delictivas que operan como respuesta a la presión por alcanzar determinado nivel de status o riqueza.

(c) Ritualismo: esta categoría agrupa trayectorias en las cuales la búsqueda de las metas mejor jerarquizadas es dejada de lado, dándose en simultáneo un apego casi compulsivo a las normas institucionales (1995a, p. 229). De esta forma, se produce a la vez una baja permanente de aspiraciones junto a ( $\mathrm{y}$ a una defensa de) un cumplimiento estricto de los preceptos morales y procedimentales del orden social.

(d) Retraimiento: la modalidad de 'retraimiento' se presenta como la menos común de las cinco en términos de frecuencia de ocurrencia, debiendo considerarse a quienes la llevan adelante como individuos que están fuera de la sociedad, pues no serían plenamente miembros al no adherir ni a los fines (que no pudieron alcanzarse) ni a los medios (que no conducían a fines buscados).

(e) Rebelión: a diferencia de las anteriores, esta categoría no supone un esfuerzo para acomodarse dentro de la estructura, sino que por el contario conlleva la intensión de desplazar la estructura social existente para instituir otra que pueda ser compartida y aceptada por el grupo o sociedad.

Establecida la tipología, Merton concluye -con relación a su preocupación sobre la desviaciónque la sociedad estadounidense, centrada exclusivamente en la valoración de los resultados de la competencia (y no en las satisfacciones de su desarrollo), y más precisamente, en los resultados monetarios de ella, produce una tendencia (una presión) hacia la anomia y a conductas desviadas como consecuencia de tal estructura valorativa.

Pasamos ahora al segundo de los textos de este autor. En Funciones manifiestas y latentes, Merton (1995b) comienza con un análisis del uso y acepciones de la palabra 'función'. Su objetivo es poder clarificar principios metodológicos para el análisis funcional, los cuales requieren claridad respecto al contenido del término 'función'.

Dejando de lado una diversidad de matices difusos que se relacionan con la idea de función (que

Social Structure and Science (1996), "Social Structure and Anomie" (pp. 132-152), Chicago University Press, refiere siempre a 'American culture', y 'Americans' para los afectados, por lo que 'estadounidense' y 'estadounidenses' resultan más precisos que 'norteamericana' y 'norteamericanos'. 
pueden remitir a fines o a meras relaciones, a situaciones objetivas o disposiciones subjetivas, a consecuencias o a meras actividades) retoma tres postulados que, a su criterio, marcaron al análisis funcional sin ser por otro lado necesarios para el mismo. Estos postulados son:

- unidad funcional: el primero, que se encuentra en Radcliffe-Brown, y luego en Malinowski, afirma que cuando algo es funcional en una sociedad, lo es para toda la sociedad. A esto Merton alega que por una parte la integración de las partes de una sociedad se da según grados o niveles, y que requiere una determinación empírica en cada caso determinar en cada sociedad hasta dónde ello se manifiesta. Asimismo, se da el caso en que ciertos fenómenos sociales son funcionales para un grupo y disfuncionales para otro, lo que agrega aún más limitaciones a este supuesto de unidad funcional.

- funcionamiento universal: el segundo, sostiene que todas las manifestaciones culturales o sociales llevan de suyo asociada alguna función vital para la sociedad. Merton no ve necesario este supuesto, señalando que puede conducir a buscar funciones que de manera indirecta o general puedan asociarse a ciertas actividades, incluso si ello no agrega ninguna claridad sobre la comprensión del fenómeno.

- indispensabilidad: con relación al anterior, se plantea la cuestión de si lo existente es necesario o no, es decir, tanto si ciertas funciones deben ser cubiertas necesariamente para la persistencia de una sociedad como si tales funciones deben corresponderse con ciertas estructuras o formaciones que las satisfacen. Este postulado se ve relativizado por el hecho de que diferentes formas funcionales cubren funciones semejantes, dando lugar a la idea de alternativas funcionales o equivalentes funcionales.

Con el objetivo de organizar el análisis funcional, Merton propone el uso de las categorías de 'manifiesto' y 'latente' para distinguir aquello que es visible a los actores -y que entra en el campo de lo 'subjetivo', en el sentido de sus motivaciones individuales- de aquello que no aparece a la conciencia de ellos, pero que sin embargo es identificable en términos de consecuencias 'objetivas' no necesariamente buscadas de la acción.

En cierto modo Merton, a través de la tematización de las cuestiones latentes, busca recuperar la atención sobre aquello menos evidente del funcionamiento social. En este sentido, orientar el análisis funcional sobre las funciones latentes (y la idea misma de análisis 'funcional') refleja una pretensión de distanciamiento con percepciones preexistentes (a cada investigación) de los fenómenos, efecto que se muestra bajo diferentes rótulos en las palabras de Merton, pero también en varias iniciativas sociológicas. Lo que aquí aparece como un llamado a estudiar no solamente las 'funciones manifiestas', también se vincula con la idea de relativizar la importancia de las motivaciones subjetivas con relación a las consecuencias objetivas que abundan en los clásicos, así como la voluntad de separar los resultados de la investigación social de términos y explicaciones del 'sentido común' (así como de 'juicios morales ingenuos' en palabras de Merton).

Para profundizar en torno a la idea de funcionales manifiestas y latentes, analiza la interacción de la 'máquina política' con el aparato de gobierno formal-legal, explicitando los mecanismos y 
complementariedades a través de los cuales una lógica aparentemente ilegítima e impropia al sistema republicano puede perpetuarse gracias a cubrir necesidades funcionales del sistema no resueltas por otras estructuras.

En el artículo, el autor abunda en ejemplos por medio de los cuales ilustra y clarifica sus posiciones teóricas; en este sentido, si bien enumerarlos aquí excede a la presentación abreviada de la obra, cabe señalar que los mismos son variados y decisivos a la hora de sustentar las apreciaciones que el texto presenta.

\section{Discusión}

La conexión de ambos autores se hará desde su contacto en común por lo que autores posteriores clasificaron como teoría del rol o de los sistemas de papeles.

\section{Teoría del rol}

En primer lugar, cabe señalar que tanto Boudon (1981) como Deutsch y Krauss (1997) vinculan a estos autores a través del tema de los 'roles' o 'papeles' en la explicación social.

Deutsch y Krauss (1997), al presentar a la psicología social como campo científico, la dividen en cinco grandes orientaciones, dentro de las cuales una es la 'teoría de rol'4. De esta teoría, destacan tres autores centrales: Mead, Merton y Goffman. No haremos referencia al primero debido a que no forma parte de la relación de autores referida en este artículo.

Sobre Merton, retoman el desarrollo sobre grupos de pertenencia y grupos de referencia y la identificación de las 'metas culturales' y los 'medios institucionalizados' para alcanzarlos. Sobre los comportamientos desviados, destacan la tipología de 'conformista', 'innovador' y 'ritualista', en función de si aceptan metas y medios, sólo metas o sólo medios respectivamente.

El 'rol' como la posición que el sujeto ocupa ( $y$, especialmente, el que percibe que ocupa) es acompañado en Merton por la preocupación por los 'grupos' en términos de colectivos de identificación y pertenencia. En este sentido, la claridad aportada por Merton -explícitamente en Merton (1964), pero también en Merton (1995a) - gira a su vez en torno a la operatoria de unos sujetos que responden (al menos analíticamente) a una lógica por la cual adoptan en primer lugar un papel, un lugar social, a partir del cual se dedican luego a operar adaptativamente desde ese papel para satisfacerlo -en términos típicos- de modo ajustado a las expectativas a las que este papel o rol se asocia. De esta forma, quien fija su pertenencia por ejemplo con relación a un campo académico (rol, grupo de referencia y eventualmente de pertenencia) manejará sus expectativas de retribución económica y de reconocimiento en forma acorde. En este sentido, los trabajos sobre grupo de Merton tienen relación directa con el problema de los roles socialmente construidos.

Goffman, según estos autores, forma parte del mismo conjunto de preocupaciones, en tanto aporta a la teoría de los roles precisiones sobre las técnicas de los actores para mostrar su rol, así como sobre los conflictos en los que dirime la atención del auditorio al igual que su propio éxito social.

\footnotetext{
${ }^{4}$ Siendo las demás: la teoría de la Gestalt, la teoría del campo, las teorías del refuerzo y la teoría psicoanalítica.
} 
Respecto a los roles y los grupos, Goffman trabaja en dos niveles la cuestión colectiva de las actuaciones: en el nivel de los grupos concretos, habla de personas que accionan cooperativamente en equipos; en el nivel de los grupos analíticos (personas que actúan de igual modo por cumplir un mismo conjunto de tareas típicas, pero que no tienen necesariamente conocimiento personal unos de otros en sus actuaciones) refiere al término 'rol'. A este respecto indica:

Si definimos el rol social como el desempeño de los derechos y de las obligaciones correspondientes a un status, podemos decir que abarcará uno o más partes y que cada una de ellas puede ser presentada por el actor en una serie de ocasiones, antes los mismos tipos de público o ante uno solo integrado por las mismas personas.

(Goffman, 1971, p. 16)

Deutsch y Krauss reponen de Goffman la importancia en los roles no sólo de cubrir requisitos funcionales, sino también sobre dar 'la apariencia' de cumplir con los requisitos del rol como su aporte más singular.

Por su parte, Boudon define al 'análisis funcional' (diferenciándolo del 'funcionalismo') como el "análisis de los sistemas de interacción que adoptan la forma de sistema de papeles"5 $\left(1981\right.$, p. 88) ${ }^{6}$. En este sentido, destaca también el carácter flexible y particularmente contradictorio de los papeles, apoyándose en argumentos de Merton para esto:

Las normas adheridas a los papeles suelen ser, según ha demostrado Merton, contradictorias. Así, el papel del investigador implica que su titular se halle dispuesto a poner sus resultados a la disposición de sus iguales tan pronto como le sea posible; pero también implica que no ha de mostrar demasiada precipitación por publicar un artículo. Del mismo modo, el investigador ha de ser impermeable a las modas intelectuales; pero también debe ser receptivo a las ideas nuevas y no sentirse obsesionado por la responsabilidad -que, por otra parte, le está también confiadade mantener las tradiciones intelectuales. Debe dejar a los demás el cuidado de apreciar el valor de los resultados que crea haber obtenido. Pero también debe defender sus hipótesis y resultados.

(Boudon 1981, p. 69)

En este sentido, presenta los papeles de los 'actores' como dotados de variabilidad, de carácter compuesto (armados de varios subroles) y dotados de ambivalencia en las normas que los definen. Asimismo, es común y esperable que el hecho de que un individuo cubra varios papeles produzca interferencias en la posibilidad de un cumplimiento estricto de los mismos. De estas características,

\footnotetext{
5 Dicen Boudon: "se pasa del análisis funcional al funcionalismo cuando se introduce ese postulado según el cual todo sistema de interacción es un sistema de papeles" (1981, p. 88)

${ }^{6}$ Esta posición no es necesariamente la de Merton; sin embargo, al referirse Merton a qué compete al análisis funcional, se responde: "Todo el campo de datos sociológicos puede someterse, y gran parte de él fue sometido, a análisis funcional. El requisito fundamental es que el objetivo de análisis represente una cosa estandarizada (es decir normada y reiterativa), tales como papeles sociales, normas institucionales, procesos sociales, normas culturales, emociones culturalmente normadas, normas sociales, instrumentos de control social, etcétera" (Merton 1995b, p. 125). Es decir, que si bien no se remite exclusivamente a sistemas de papeles, sí coincidiría Merton en excluir aquellos sistemas de interacciones no estructuradas a favor de aquellos organizados por medio de formas pautadas estables.
} 
se deriva que hay un margen de autonomía intrínseca a los papeles, tal como cree conveniente definirlos en el marco de una teoría de los roles que reúna los trabajos sociológicos que aportan en esa dirección.

Si bien Boudon no emprende una caracterización de influencia por autor en una teoría funcional de los roles, recurre a Goffman para ilustrar el carácter no previsible (o sólo gradualmente cognoscible) de los papeles para quienes los desempeñan: refiere para ello a un relato de Goffman en el libro Asilos donde se narra cómo un pariente próximo convence a un sujeto de concurrir a la 'ayuda' médica, sin advertir que ni bien se encuentre en la situación de consultorio en la que los tres estén reunidos, él (el pariente) no operará (como él supuso y prometió) como garante de la libertad del paciente, sino como cómplice silencioso de la mirada 'objetiva' del médico (tanto médico como familiar se encuentran fuera de la visión 'perturbada' del paciente, y esto los ligará irremediablemente) (Boudon, 1981, p. 86).

Además de este carácter gradual del descubrimiento de la naturaleza e implicancias de los roles en los que las personas se embarcan, Goffman facilita numerosas explicaciones sobre los funcionamientos de la personificación de papeles sociales, tales que su articulación grupal, su organización diferenciada de saberes, la separación entre el público y los ejecutores de la actuación, entre otras.

Esta caracterización extiende y complementa las observaciones de Merton sobre las presiones recibidas según posición social en la antesala de las relaciones desviadas, así como con su interés por resaltar el margen de libertad promovido por el carácter contradictorio de los papeles.

\section{Conclusiones}

A modo de cierre, puede sostenerse que ambos autores han dado por supuesto, desde diferentes perspectivas, y a la vez extendido el concepto de rol y de sistemas de roles a partir de sus investigaciones y aportes teórico-metodológicos. Analizar un sistema de roles es una tarea compleja y específica en términos de relación entre los actores, entre los roles, y de los actores con los roles.

En el caso de Goffman, la exploración de la estructuración de la dinámica de las interacciones cotidianas es el tema más saliente, especificándose caracteres generales de la interacción antes inadvertidos o no tan claramente sistematizados, tales que la separación entre 'backstage' y escena, el uso casi-simultáneo de discursos y roles para ambos espacios, la tarea ineludible del manejo individual y grupal de la impresión de la propia imagen sobre los demás, así como la exigencia social sobre los actores de sostener una cantidad enorme de aspectos formales para lograr sostener el parecer de cada rol e institución social.

En Merton, se destacan -también en el campo de la mirada de los actores- la voluntad de observar las funciones que ellos cumplen -siendo o no conscientes de hacerlo- y al mismo tiempo las metas y restricciones que el sistema social les propone como posibles en virtud de los papeles y lugares que ellos ocupan.

En ambos casos, la interacción de lo institucionalizado con la subjetividad es problemática, reservándose márgenes de libertad a los actores al tiempo que se destaca el carácter mediado, 
simbólico y socialmente estructurado de las posibilidades de maniobra disponibles.

En ese marco, los elementos teóricos aquí recuperados sirven como herramientas para el estudio de la interacción, señalando ejes de análisis clave: ¿cómo se articulan los medios admisibles con los fines legitimados? ¿Qué 'equipos' intervienen en la acción? ¿Qué estrategias y tácticas ponen en juego para controlar su imagen y la de los otros? ¿Qué efectos tienen éstas en la dinámica colectiva de la interacción?

Los desajustes, inconsistencias, conflictos, imposibilidades y tensiones entre roles y actores concretos se sugieren como constitutivos del funcionamiento de lo social, y pueden en tanto tales ser asumidos y precisados en toda construcción de explicaciones que den cuenta de las dinámicas y lógicas de la interacción social.

\section{Referencias bibliográficas}

Boudon, R. (1981). La lógica de lo social. Madrid: Rialp.

Cole, S. (2004). Merton's Contribution to the Sociology of Science. Social Studies of Science, Vol. 34 (6), pp. 829-844.

Collins, R. (1986). The Passing of Intellectual Generations: Reflections on the Death of Erving Goffman. Sociological Theory, Vol. 4 (1), pp. 106-113.

Crothers, C. (1987). Merton's intellectual biography. En Robert K. Merton, Londres: Taylor \& Francis, pp. 23-50.

Deutsch, M. y Krauss, R. (1997). Teorías en Psicología Social. Paidós, Barcelona

Goffman, E. (1971). La presentación de la persona en la vida cotidiana, Buenos Aires: Amorrortu editores.

Goffman, E. (1974). Frame Analysis, Nueva York: Harper.

Merton, R. K. (1964). Teoría y estructuras sociales. México: Fondo de Cultura Económica.

Merton, R. K. (1995a). Estructura social y anomia. En Teoría y estructuras sociales, Fondo de Cultura Económica, pp. 209-239.

Merton, R. K. (1995b). Funciones manifiestas y latentes. En Teoría y estructuras sociales, Fondo de Cultura Económica, pp. 92-160.

Ritzer, G. (2002). Teoría sociológica moderna. Madrid: McGraw Hill. 